\title{
INPUT RETRIEVAL IN FINITE DIMENSIONAL LINEAR SYSTEMS
}

\author{
P. G. HOWLETT \\ (Received 18 November 1980) \\ (Revised 1 March 1981)
}

\begin{abstract}
For finite dimensional linear systems it is known that in certain circumstances the input can be retrieved from a knowledge of the output only. The main aim of this paper is to produce explicit formulae for input retrieval in systems which do not possess direct linkage from input to output. Although two different procedures are suggested the fundamental idea in both cases is to find an expression for the inverse transfer function of the system. In the first case this is achieved using a general method of power series inversion and in the second case by a sequence of elementary operations on a Rosenbrock type system matrix.
\end{abstract}

\section{Introduction}

The first systematic paper on the problem of input retrieval in finite dimensional linear systems appears to have been the one by Brockett and Mesarovic [1] in which conditions were given that are necessary and sufficient to guarantee retrieval of input from a knowledge of the output only. Subsequently these conditions were modified by Sain and Massey [3] and later by Wang and Davison [5] to produce alternative simplified conditions. Methods for input retrieval were first suggested by Sain and Massey [3] and Silverman [4]. Since that time many other authors have made significant contributions to the subject. The present paper is motivated by the methods of Sain and Massey [3] in which the retrieval problem is formulated via a collection of matrix equations. Some of the questions posed by this formulation appear not to have been answered.

(c) Copyright Australian Mathematical Society 1982 
In this paper we will consider only systems which can be written in the form

$$
\left.\begin{array}{l}
\dot{x}(t)=A x(t)+B u(t) \\
y(t)=C x(t)
\end{array}\right\}
$$

with $x(0)=\theta$ where $x \in R^{n}, u \in R^{m}, y \in R^{m}$ and $n \geqslant m$. Further, $A, B$ and $C$ are real matrices of appropriate dimensions. Throughout this paper, $\theta$ will denote a matrix of appropriate dimensions with zero elements. We will assume that both $B$ and $C$ have rank $m$ and that $u(t)$ is analytic "near" $t=0$ - to which neighbourhood the analysis will be restricted. The problem is to state conditions under which the input $u(t)$ can be retrieved from a knowledge of the output $y(t)$ only and to explain the structure of a suitable recovery procedure. The omission of direct feed from input to output is deliberate because for many commonly formulated systems there is no such connection and because the inclusion of such a term tends to obscure a direct retrieval procedure for these systems.

In order to present the problem in its simplest form we will for the moment assume that the product matrix $C B$ is invertible. By taking a formal Laplace transform of (1) we have

$$
\bar{y}(s)=T(s) \bar{u}(s)
$$

where $\bar{u}(s)$ and $\bar{y}(s)$ are the respective Laplace transforms of $u(t)$ and $y(t)$. The transfer function $T(s)$ is given by the formula

$$
\begin{aligned}
T(s) & =C\left(s I_{n}-A\right)^{-1} B \\
& \left.=(1 / s) \sum_{j=0}^{\infty} C A^{j} B / s^{j} \quad \text { (for sufficiently large }|s|\right) \\
& =(1 / s) \sum_{j=0}^{\infty} J_{j} / s^{j}=(1 / s) J(1 / s),
\end{aligned}
$$

in which we have used $J_{j}=C A^{j} B$ for each $j=0,1,2, \ldots$ Since it is easy to see that $\left\|J_{j}\right\|<a^{j+1}$ for some sufficiently large real constant $a>0$, it follows that $T(s)$ is analytic in the region $|s|>a$. In attempting to solve (2) it seems intuitively reasonable to seek a "solution" in the form

$$
\bar{u}(s)=[T(s)]^{-1} \bar{y}(s)
$$

where the inverse transfer function $[T(s)]^{-1}$ is defined as nearly as possible by a power series in $1 / s$. In fact if we assume an expansion of the form

$$
[T(s)]^{-1}=s \sum_{j=0}^{\infty} K_{j} / s^{j}=s K(1 / s),
$$


then it follows by equating coefficients that the $K_{j}$ must satisfy each of the countable collections of equations

$$
J_{0} K_{0}=I_{m} \quad \text { and } \quad \sum_{j=0}^{k} J_{k-j} K_{j}=\theta \quad \text { for } k=1,2,3, \ldots
$$

and

$$
K_{0} J_{0}=I_{m} \quad \text { and } \quad \sum_{j=0}^{k} K_{j} J_{k-j}=\theta \quad \text { for } k=1,2,3, \ldots
$$

We will show that there is a uniquely defined solution to (6) and that the coefficients $K_{j}$ are sufficiently small to ensure that $[T(s)]^{-1}$ is well defined by (5). Because the $K_{j}$ can be shown to satisfy a finite recurrence relation it is necessary to solve only a finite subcollection of the equations (6). Nevertheless the suggested technique of direct power series inversion does not generate the simplest recurrence satisfied by the $K_{j}$ and for this reason we also consider an alternative method of retrieval.

Let us define a slightly modified form $N(s)$ of the Rosenbrock system matrix [2] by setting

$$
N(s)=\left[\begin{array}{cc}
\theta & -C \\
-B & s I_{n}-A
\end{array}\right] .
$$

If we choose non singular matrices $B^{\dagger}$ and $C^{\dagger}$ written in partitioned form as

$$
B^{\dagger}=\left[\begin{array}{l}
B_{1}^{\dagger} \\
B_{2}^{\dagger}
\end{array}\right] \text { and } C^{\dagger}=\left[C_{1}^{\dagger} C_{2}^{\dagger}\right]
$$

such that

$$
B^{\dagger} B=\left[\begin{array}{c}
I_{m} \\
\theta
\end{array}\right] \text { and } C C^{\dagger}=\left[\begin{array}{ll}
I_{m} & \theta
\end{array}\right]
$$

then using elementary row and column operations on $N(s)$ we can show that it is (rank) equivalent to each of the matrices

$$
N_{1}(s)=\left[\begin{array}{cc}
-C\left(s I_{n}-A\right)^{-1} B & \theta \\
\theta & s I_{n}-A
\end{array}\right]
$$

and

$$
N_{2}(s)=\left[\begin{array}{ccc}
I_{m} & \theta & \theta \\
\theta & I_{m} & \theta \\
\theta & \theta & B_{2}^{\dagger}\left(s I_{n}-A\right) C_{2}^{\dagger}
\end{array}\right]
$$


From this equivalence it follows that $T(s)=C\left(s I_{n}-A\right)^{-1} B$ is invertible if and only if $B_{2}^{\dagger}\left(s I_{n}-A\right) C_{2}^{\dagger}$ is invertible and hence we obtain the key inversion formula

$$
[T(s)]^{-1}=B_{1}^{\dagger}\left(s I_{n}-A\right)\left\{\left(s I_{n}-A\right)^{-1}-C_{2}^{\dagger}\left[B_{2}^{\dagger}\left(s I_{n}-A\right) C_{2}^{\dagger}\right]^{-1} B_{2}^{\dagger}\right\}\left(s I_{n}-A\right) C_{1}^{\dagger}
$$

If we assume that the product matrix $C B$ is invertible then we can use (7) to show that for $j=2,3,4, \ldots$ the coefficients $K_{j}$ of the previous section satisfy a recurrence relation defined by the minimum polynomial of the matrix $\left(B_{2}^{\dagger} C_{2}^{\dagger}\right)^{-1} B_{2}^{\dagger} A C_{2}^{\dagger}$. It is also possible to rewrite (7) in the form

$$
[T(s)]^{-1}=K_{0} s+K_{1}+H\left(s I_{n-m}-F\right)^{-1} G
$$

and hence part of the input retrieval can be achieved via an "inverse" system in the form of (1).

\section{On the inversion of power series-non singular case.}

In order to discuss the construction of the inverse transfer function $[T(s)]^{-1}$ using a power series in $1 / s$ it is convenient first to consider the general problem of power series inversion. Because of the repeated use of a particular type of augmented matrix we will use the following notation throughout. If $\left\{A_{j}\right\}$ is a sequence of $(m \times m)$ matrices then for each $k=1,2,3, \ldots$ we define a sequence $\left\{\mathbb{Q}_{j}^{(k)}\right\}$ of $(m k \times m k)$ matrices as follows. Let

$$
\mathbb{Q}_{0}^{(k)}=\left[\begin{array}{cccc}
A_{0} & \theta & \cdots & \theta \\
A_{1} & A_{0} & \cdots & \theta \\
\vdots & \vdots & & \vdots \\
A_{k-1} & A_{k-2} & \cdots & A_{0}
\end{array}\right]
$$

and

$$
\mathbb{Q}_{j}^{(k)}=\left[\begin{array}{cccc}
A_{j k} & A_{j k-1} & \cdots & A_{(j-1) k+1} \\
A_{j k+1} & A_{j k} & \cdots & A_{(j-1) k+2} \\
\vdots & \vdots & & \vdots \\
A_{(j+1) k-1} & A_{(j+1) k-2} & \cdots & A_{j k}
\end{array}\right]
$$

for each $j=1,2,3, \ldots$ 
THEOREM 1. Let $\left\{A_{j}\right\}$ be a sequence of square $(m \times m)$ matrices such that $A_{0}$ is non singular. Then the linear systems

$$
A_{0} X_{0}=I_{m} \quad \text { and } \quad \sum_{j=0}^{k} A_{k-j} X_{j}=\theta \quad \text { for } k=1,2,3, \ldots
$$

and

$$
Y_{0} A_{0}=I_{m} \quad \text { and } \quad \sum_{j=0}^{k} Y_{j} A_{k-j}=\theta \quad \text { for } k=1,2,3, \ldots
$$

each have uniquely defined solutions $\left\{X_{j}\right\}$ and $\left\{Y_{j}\right\}$ respectively and furthermore $X_{j}=Y_{j}$ for all $j=0,1,2, \ldots$.

Proof. We can define solutions to (9) using the following inductive procedure

$$
X_{0}=A_{0}^{-1} \quad \text { and } \quad X_{k}=-A_{0}^{-1}\left(\sum_{j=0}^{k-1} A_{k-j} X_{j}\right) \quad \text { for } k=1,2,3, \ldots
$$

and

$$
Y_{0}=A_{0}^{-1} \quad \text { and } \quad Y_{k}=-\left(\sum_{j=0}^{k-1} Y_{j} A_{k-j}\right) A_{0}^{-1} \quad \text { for } k=1,2,3, \ldots
$$

It is easy to see that these solutions are unique. If we now choose an arbitrary value of $k$, say $k=i$ then we can see that

$$
\mathbb{Q}_{0}^{(i)} \mathfrak{X}_{0}^{(i)}=I_{i m} \quad \text { and } \quad \mathscr{Y}_{0}^{(i)} \mathbb{Q}_{0}^{(i)}=I_{i m} .
$$

Thus from elementary matrix algebra $\mathcal{X}_{0}^{(i)}=\mathscr{Q}_{0}^{(i)}$ and the proof is complete.

Corollary 1. Let $\left\{A_{j}\right\}$ be a sequence of square matrices as in Theorem 1 and suppose also that $\left\|A_{j}\right\|<a^{j+1}$ for some real number $a>0$. Then the function $A(s)=\sum_{j=0}^{\infty} A_{j} s^{j}$ is well defined for $|s|<1 / a$ and we can find a uniquely determined series $X(s)=\sum_{j=0}^{\infty} X_{j} s^{j}$ with positive radius of convergence and such that in the region where both series converge we have $A(s) X(s)=X(s) A(s)=I_{m}$. We write $X(s)=[A(s)]^{-1}$.

NotE: In all cases $\|A\|=\sup _{x \in R^{n}}\|A x\| /\|x\|$ where $\|x\|^{2}=\sum_{i=1}^{n} x_{i}^{2}$.

Proof. Define $\left\{X_{j}\right\}$ as in Theorem 1 and let

$$
b=\max \left\{\left\|A_{0}^{-1}\right\|,\left\|A_{0}^{-1}\right\| a^{2}+a\right\}+1 .
$$

It is now easy to see that $\left\|X_{j}\right\|<b^{j+1}$ for all $j=0,1,2, \ldots$ 
Corollary 2. Let $\left\{A_{j}\right\}$ be defined as in Theorem 1 and suppose that for some integer $r>0$ we have

$$
A_{j+r+1}=\sum_{k=1}^{r+1} \alpha_{k} A_{j+r+1-k} \text { for each } j=0,1,2, \ldots
$$

If we define a finite sequence $\left\{A_{j}^{*}\right\}$ by setting

$$
A_{0}^{*}=A_{0}, A_{j}^{*}=A_{j}-\sum_{k=1}^{j} \alpha_{k} A_{j-k} \quad \text { for each } j=1,2, \ldots, r,
$$

and use the notation $A^{*}(s)=\sum_{j=0}^{r} A_{j}^{*} s^{j}$, then

$$
[A(s)]^{-1}=\left(1-\sum_{k=1}^{r} \alpha_{k} s^{k}\right)\left[A^{*}(s)\right]^{-1} \text {. }
$$

TheOREM 2. Let $A(s)=\sum_{j=0}^{r} A_{j} s^{j}$ where $\left\{A_{j}\right\}$ is a finite sequence of square $(m \times m)$ matrices with $A_{0}$ non singular. Then we can write $[A(s)]^{-1}=\sum_{j=0}^{\infty} X_{j} s^{j}$ where the $X_{j}$ satisfy a finite recurrence relation of the form

$$
X_{j+r h}=\sum_{k=1}^{h} \xi_{k} X_{j+r h-r k} \text { for each } j=0,1,2, \ldots, \text { where } h \leqslant r m .
$$

PRoof. Define $\left\{X_{j}\right\}$ as in Theorem 1 and let $\mathcal{L}=\mathbb{Q}_{0}^{(r)}$ and $\mathscr{Q}=\mathbb{Q}_{1}^{(r)}$. Then for each $i=1,2,3, \ldots$ we have

$$
\left[\begin{array}{ccccc}
\mathcal{E} & \theta & \theta & \cdots & \theta \\
\mathcal{U} & \mathcal{E} & \theta & \cdots & \theta \\
\theta & \mathcal{Q} & \mathcal{E} & \cdots & \theta \\
\vdots & \vdots & \vdots & & \vdots \\
\theta & \theta & \theta & \cdots & \mathcal{E}
\end{array}\right]\left[\begin{array}{c}
\mathcal{X}_{0}^{(r)} \\
\mathcal{X}_{1}^{(r)} \\
\mathcal{X}_{2}^{(r)} \\
\vdots \\
\mathcal{X}_{i}^{(r)}
\end{array}\right]=\left[\begin{array}{c}
I_{r m} \\
\theta \\
\theta \\
\vdots \\
\theta
\end{array}\right] .
$$

Since $A_{0}$ is non singular it follows that $\mathcal{L}$ is non singular and hence we can solve these equations to give

$$
\mathfrak{X}_{j}^{(r)}=(-1)^{j}\left(\mathfrak{e}^{-1} \mathcal{Q}\right)^{j} \mathfrak{e}^{-1} \text { for } j=0,1,2, \ldots, i .
$$

Since $i$ is arbitrary it follows that this formula holds for all $j$. Thus the $\mathscr{X}_{j}^{(r)}$ satisfy a finite recurrence relation defined by the minimum polynomial of the $m r \times m r$ matrix $(-1) \mathfrak{L}^{-1} \mathcal{Q}$. Therefore for some fixed $h \leqslant m r$ we have

$$
\mathfrak{X}_{j+h}^{(r)}=\sum_{k=1}^{h} \xi_{k} \mathscr{X}_{j+h-k}^{(r)} \text { for } j=0,1,2, \ldots
$$


By applying this recurrence to each position in the partitioned matrix we obtain

$$
X_{j+r h}=\sum_{k=1}^{h} \xi_{k} X_{j+r h-r k} \text { for } j=0,1,2, \ldots
$$

One practical disadvantage of this procedure is that the recurrence relation derived for the coefficients $X_{j}$ is not (usually) the simplest such recurrence.

\section{On the inversion of power series-singular case.}

We will continue the discussion of the previous section by extending the results obtained in that section to cover inversion of a power series $A(s)=\sum_{k=0}^{\infty} A_{k} s^{k}$ in the case where $A_{0}$ is singular.

THEOREM 3. Let $\left\{A_{k}\right\}$ be a sequence of square $(m \times m)$ matrices such that $A_{0}$ is singular. If we can find non singular matrices $M$ and $N$ such that

$$
M A_{0} N=\left[\begin{array}{c:c}
I_{m_{1}} & \theta \\
\hdashline \theta & \bar{\theta}
\end{array}\right] \text { and } M A_{1} N=\left[\begin{array}{ccc}
A_{111}^{*} & A_{112}^{*} \\
\hdashline A_{121}^{*} & I_{m_{2}}
\end{array}\right]
$$

where $m_{1}, m_{2}>0$ and $m_{1}+m_{2}=m$, then the linear systems

$$
A_{0} X_{0}=\theta, \quad A_{1} X_{0}+A_{0} X_{1}=I_{m} \quad \text { and } \quad \sum_{j=0}^{k} A_{k-j} X_{j}=\theta \quad \text { for } k=2,3, \ldots
$$

and

$$
Y_{0} A_{0}=\theta, \quad Y_{0} A_{1}+Y_{1} A_{0}=I_{m} \quad \text { and } \quad \sum_{j=0}^{k} Y_{j} A_{k-j}=\theta \quad \text { for } k=2,3, \ldots
$$

each have uniquely defined solutions $\left\{X_{j}\right\}$ and $\left\{Y_{j}\right\}$ respectively and furthermore $X_{j}=Y_{j}$ for all $_{j}=0,1,2, \ldots$.

Proof. Choose $M$ and $N$ as stated and define

$$
A_{j}^{*}=M A_{j} N, \quad X_{j}^{*}=N^{-1} X_{j} M^{-1} \quad \text { and } \quad Y_{j}^{*}=N^{-1} Y_{j} M^{-1} .
$$

It is clear that for all $k$ and any scalar $\alpha_{k}$ we have

$$
\sum_{j=0}^{k} A_{k-j} X_{j}=\alpha_{k} I_{m} \Leftrightarrow \sum_{j=0}^{k} A_{k-j}^{*} X_{j}^{*}=\alpha_{k} I_{m}
$$

and

$$
\sum_{j=0}^{k} Y_{j} A_{k-j}=\alpha_{k} I_{m} \Leftrightarrow \sum_{j=0}^{k} Y_{j}^{*} A_{k-j}^{*}=\alpha_{k} I_{m}
$$


Thus the original systems (13) have solutions if and only if the modified systems (where $A_{j}$ is replaced by $A_{j}^{*}$ ) have solutions. Moreover there is a 1-1 correspondence between the solutions to the original equations and the solutions to the modified equations. Thus without loss of generality we assume

$$
A_{0}=\left[\begin{array}{c:c}
I_{m_{1}} & \theta \\
\hdashline \theta & \theta
\end{array}\right] \text { and } A_{1}=\left[\begin{array}{l:l}
A_{111} & A_{112} \\
\hdashline A_{121} & I_{m_{2}}
\end{array}\right] \text {. }
$$

Now

$$
A_{0} X_{0}=\theta \Rightarrow X_{011}=\theta \text { and } X_{012}=\theta
$$

and

$$
A_{1} X_{0}+A_{0} X_{1}=I_{m} \Rightarrow X_{021}=\theta \text { and } X_{022}=I_{m_{2}} .
$$

Thus we obtain a unique solution for $X_{0}$, namely

$$
X_{0}=\left[\begin{array}{cc}
\theta & { }^{\prime} \\
\bar{\theta} & +\bar{I}_{m_{2}}^{-}
\end{array}\right]
$$

If we now suppose that $X_{j}$ is uniquely defined for $0 \leqslant j \leqslant k-1$ then

$$
\begin{gathered}
A_{0} X_{k}=-\sum_{j=0}^{k-1} A_{k-j} X_{j} \text { implies that } \\
X_{k 11}=-\left(\sum_{j=0}^{k-1} A_{k-j} X_{j}\right)_{11} \text { and } X_{k 12}=-\left(\sum_{j=0}^{k-1} A_{k-j} X_{j}\right)_{12}
\end{gathered}
$$

and

$$
\begin{gathered}
A_{1} X_{k}+A_{0} X_{k+1}=-\sum_{j=0}^{k-1} A_{k+1-j} X_{j} \text { implies that } \\
X_{k 21}=-A_{121} X_{k 11}-\left(\sum_{j=0}^{k-1} A_{k+1-j} X_{j}\right)_{21} \text { and } \\
X_{k 22}=-A_{121} X_{k 12}-\left(\sum_{j=0}^{k-1} A_{k+1-j} X_{j}\right)_{22}
\end{gathered}
$$

Thus $X_{k}$ is uniquely defined. Hence the solution $\left\{X_{j}\right\}$ is uniquely defined and a similar argument shows that the solution $\left\{Y_{j}\right\}$ is also uniquely defined. Now for an arbitrarily chosen value of $k$, say $k=i+1$ we have

$$
\mathcal{Q}_{0}^{(i+1)} \mathscr{X}_{0}^{(i+1)}=\mathcal{g} \text { and } \mathcal{Y}_{0}^{(i+1)} \mathcal{Q}_{0}^{(i+1)}=\mathcal{g}
$$


where $g$ denotes the $(i+1) m \times(i+1) m$ matrix

$$
g=\left[\begin{array}{cccccc}
\theta & \theta & \theta & \cdots & \theta & \theta \\
I_{m} & \theta & \theta & \cdots & \theta & \theta \\
\theta & I_{m} & \theta & \cdots & \theta & \theta \\
\vdots & \vdots & \vdots & & \vdots & \vdots \\
\theta & \theta & \theta & \cdots & \theta & \theta \\
\theta & \theta & \theta & \cdots & I_{m} & \theta
\end{array}\right] .
$$

Thus we have

$$
\mathscr{G} \mathfrak{X}_{0}^{(i+1)}=\mathscr{Y}_{0}^{(i+1)} \mathbb{Q}_{0}^{(i+1)} \mathscr{X}_{0}^{(i+1)}=\mathcal{Y}_{0}^{(i+1)} \mathcal{g} .
$$

It follows that $\mathfrak{X}_{0}^{(i)}=\mathscr{Y}_{0}^{(i)}$.

\section{LEMMA 1. For the partitioned matrix}

$$
A=\left[\begin{array}{l:l}
A_{11} & A_{12} \\
\hdashline A_{21} & A_{22}
\end{array}\right]
$$

we have $\left\|A_{p q}\right\| \leqslant\|A\| \leqslant \Sigma_{p, q}\left\|A_{p q}\right\|$.

COROLlary 3. Let $\left\{A_{k}\right\}$ be a sequence of matrices as in Theorem 3 and suppose in addition that $\left\|A_{k}\right\|<a^{k+1}$ for some real number $a>0$. Then the function $A(s)=\sum_{k=0}^{\infty} A_{k} s^{k}$ is well defined for $|s|<1 / a$ and we can find a uniquely determined series $X(s)=\sum_{\vec{k}=0} X_{k} s^{k}$ with positive radius of convergence and such that in the region where both series converge we have $A(s) X(s)=X(s) A(s)=s I_{m}$. We write $[A(s)]^{-1}=X(s) / s$ for $s \neq 0$.

Proof. As in Theorem 3 we assume

$$
A_{0}=\left[\begin{array}{c:c}
I_{m_{1}} & \theta \\
\hdashline \theta & \theta
\end{array}\right] \text { and } A_{1}=\left[\begin{array}{c:c}
A_{111} & A_{112} \\
\hdashline A_{121} & I_{m_{2}}
\end{array}\right]
$$

and we define $\left\{X_{j}\right\}$ as in the proof of Theorem 3. Let

$$
b=\max \left\{6 a^{4}+a, 6 a^{2}+a, 1\right\}+1 .
$$

It is now easy to use Lemma 1 to show that $\left\|X_{j}\right\|<b^{j+1}$ for each $j=0,1,2, \ldots$

THEOREM 4. Let $A(s)=\sum_{j=0}^{r} A_{j} s^{j}$ where $\left\{A_{j}\right\}$ is a finite sequence of square $(m \times m)$ matrices satisfying the conditions of Theorem 3 . Then we can write

$$
[A(s)]^{-1}=X(s) / s \text { for } s \neq 0 \text {, }
$$


where $X(s)=\sum_{j=0}^{\infty} X_{j} s^{j}$ and the $X_{j}$ satisfy a finite recurrence relation of the form

$$
X_{j+r h}=\sum_{k=1}^{h} \xi_{k} X_{j+r h-r k} \quad \text { for each } j=0,1,2, \ldots \text { where } h \leqslant r m .
$$

Proof. Once again we assume

$$
A_{0}=\left[\begin{array}{c:c}
I_{m_{1}} & \theta \\
\hdashline \theta & \theta
\end{array}\right] \text { and } A_{1}=\left[\begin{array}{c:c}
A_{111} & A_{112} \\
\hdashline A_{121} & I_{m_{2}}
\end{array}\right]
$$

and define $\left\{X_{j}\right\}$ as in Theorem 3. If we now define

$$
B_{j}=\left[\begin{array}{ll}
A_{j+1,22} & A_{j 21} \\
A_{j+1,12} & A_{j 11}
\end{array}\right] \text { and } W_{j}=\left[\begin{array}{ll}
X_{j 21} & X_{j 22} \\
X_{j+1,11} & X_{j+1,12}
\end{array}\right]
$$

for each $j=0,1,2, \ldots$ and if we let $\mathcal{L}=\mathscr{B}_{0}^{(r)}$ and $\mathscr{U}=\mathscr{B}_{1}^{(r)}$ then for each $i=1,2,3, \ldots$ we have

$$
\left[\begin{array}{ccccc}
\mathcal{L} & \theta & \theta & \cdots & \theta \\
\mathcal{U} & \mathcal{E} & \theta & \cdots & \theta \\
\theta & \mathcal{Q} & \mathcal{L} & \cdots & \\
\vdots & \vdots & \vdots & & \vdots \\
\theta & \theta & \theta & \cdots & \mathcal{E}
\end{array}\right]\left[\begin{array}{c}
\mathcal{W}_{0}^{(r)} \\
\mathcal{W}_{1}^{(r)} \\
\mathcal{W}_{2}^{(r)} \\
\vdots \\
\mathcal{W}_{i}^{(r)}
\end{array}\right]=\left[\begin{array}{c}
\mathcal{K} \\
\theta \\
\theta \\
\vdots \\
\theta
\end{array}\right],
$$

where the matrix $\mathcal{K}$ is defined by

$$
\mathscr{K}=\left[\begin{array}{cccccccc}
\theta & I_{m_{2}} & \theta & \theta & \theta & \cdots & \theta & \theta \\
I_{m_{1}} & \theta & \theta & \theta & \theta & \cdots & \theta & \theta \\
\theta & \theta & \theta & I_{m_{2}} & \theta & \cdots & \theta & \theta \\
\theta & \theta & I_{m_{1}} & \theta & \theta & \cdots & \theta & \theta \\
\vdots & \vdots & \vdots & \vdots & \vdots & & \vdots & \vdots \\
\theta & \theta & \theta & \theta & \theta & \cdots & \theta & I_{m_{2}} \\
\theta & \theta & \theta & \theta & \theta & \cdots & I_{m_{1}} & \theta
\end{array}\right]_{r m \times r m}
$$

From (15) it is clear that for each $j=0,1,2, \ldots, i$ we have

$$
\operatorname{wos}_{j}^{(r)}=(-1)^{j}\left(\mathcal{L}^{-1} \mathcal{Q}\right)^{j} \mathcal{L}^{-1} \mathcal{K} \text {. }
$$

The remainder of the proof follows as in Theorem 2.

THEOREM 5. Let $\left\{A_{j}\right\}$ be a sequence of square $(m \times m)$ matrices such that neither the conditions of Theorem 1 nor the conditions of Theorem 3 is satisfied. Let $i$ be the 
smallest integer for which we can find non singular $\mathfrak{N}$ and $\mathscr{\Re}$ such that

$$
\mathscr{R} \mathbb{Q}_{0}^{(i)} \Re=\left[\begin{array}{c:c}
I_{m_{1}} & \theta \\
\hdashline \theta & \bar{\theta}
\end{array}\right] \quad \text { and } \quad \Re \mathbb{Q}_{1}^{(i)} \Re=\left[\begin{array}{c:c}
\mathcal{Q}_{111}^{*} & \mathbb{Q}_{112}^{*} \\
\hdashline \mathbb{Q}_{121}^{*} & I_{m_{2}}
\end{array}\right]
$$

where $m_{1}, m_{2}>0$ and $m_{1}+m_{2}=i m$. Then the linear systems

$$
\begin{gathered}
\sum_{j=0}^{k} A_{k-j} X_{j}=\theta \text { for } k=0,1,2, \ldots \text { and } k \neq i, \\
\sum_{j=0}^{i} A_{i-j} X_{j}=I_{m},
\end{gathered}
$$

and

$$
\begin{gathered}
\sum_{j=0}^{k} Y_{j} A_{k-j}=\theta \text { for } k=0,1,2, \ldots \text { and } k \neq i, \\
\sum_{j=0}^{i} Y_{j} A_{i-j}=I_{m},
\end{gathered}
$$

each have uniquely defined solutions $\left\{X_{j}\right\}$ and $\left\{Y_{j}\right\}$ respectively and furthermore $X_{j}=Y_{j}$ for all $j$.

Proof. It is easy to see that the equations (17) above can be rewritten as

$$
\begin{array}{r}
\mathbb{Q}_{0}^{(i)} \mathfrak{X}_{0}^{(i)}=\theta, \quad \mathbb{Q}_{1}^{(i)} \mathfrak{X}_{0}^{(i)}+\mathbb{Q}_{0}^{(i)} \mathfrak{X}_{1}^{(i)}=I_{i m} \quad \text { and } \quad \sum_{j=0}^{k} \mathbb{Q}_{k-j}^{(i)} \mathfrak{X}_{j}^{(i)}=\theta \\
\text { for } k=2,3, \ldots
\end{array}
$$

and

$$
\begin{aligned}
& \mathscr{Y}_{0}^{(i)} \mathbb{Q}_{0}^{(i)}=\theta, \quad \mathcal{Y}_{1}^{(i)} \mathbb{Q}_{0}^{(i)}+\mathscr{Y}_{0}^{(i)} \mathbb{Q}_{1}^{(i)}=I_{i m} \quad \text { and } \quad \sum_{j=0}^{k} \mathscr{Y}_{j}^{(i)} \mathbb{Q}_{k-j}^{(i)}=\theta \\
& \text { for } k=2,3, \ldots
\end{aligned}
$$

The required result now follows from Theorem 3 .

COROLlaRY 4. Let $\left\{A_{j}\right\}$ be a sequence of square $(m \times m)$ matrices satisfying the conditions of Theorem 5 and also with $\left\|A_{j}\right\|<a^{j+1}$ for some real $a>0$. Then $A(s)=\sum_{j=0}^{\infty} A_{j} s^{j}$ is well defined for $|s|<1 / a$ and we can find a uniquely determined series $X(s)=\sum_{j=0}^{\infty} X_{j} s^{j}$ with positive radius of convergence and such that in the region where both series converge $A(s) X(s)=X(s) A(s)=s^{i} I_{m}$. We write $[A(s)]^{-1}=X(s) / s^{i}$ for $s \neq 0$. 
Proof. Use the method of Theorem 5 and apply Corollary 3 to obtain appropriate bounds on the coefficients $X_{j}$.

THForem 6. Let $A(s)=\sum_{j=0}^{r} A_{j} s^{j}$ where $\left\{A_{j}\right\}$ is a finite sequence satisfying the conditions of Theorem 5. Then we can write

$$
[A(s)]^{-1}=X(s) / s^{i} \text { for } s \neq 0,
$$

where $X(s)=\sum_{j=0}^{\infty} X_{j} s^{j}$ and the $X_{j}$ satisfy a finite recurrence relation of the form

$$
X_{j+p i h}=\sum_{k=1}^{h} \xi_{k} X_{j+p i h-p i j} \quad(j=0,1,2, \ldots)
$$

where $h \leqslant$ pim and where $p$ is the unique integer such that $p i \geqslant r>(p-1) i$.

Proof. Use the methods of Theorem 5 and Corollary 4 and apply Theorem 4 to the finite sequence $\left\{\mathbb{Q}_{j}^{(i)}\right\}$ of $i m \times$ im matrices.

EXAMPLE. The methods of inversion used in this section will now be illustrated with a numerical example. Consider the finite sequence

$$
A_{0}=\left[\begin{array}{ll}
1 & 0 \\
0 & 0
\end{array}\right], \quad A_{1}=\left[\begin{array}{ll}
1 & 1 \\
0 & 0
\end{array}\right] \quad \text { and } \quad A_{2}=\left[\begin{array}{ll}
1 & 0 \\
0 & 1
\end{array}\right] \text {. }
$$

It can be shown that neither the conditions for Theorem 1 nor Theorem 3 hold and hence we proceed as in Theorem 5, Corollary 4 and Theorem 6. [In the appendix we will mention a convenient test due to Sain and Massey which allows us to determine which of the inversion theorems (if any) can be applied.] In this example we simply note that if we choose non singular matrices $\mathscr{R}$ and $\mathscr{R}$ given by

$$
\Re=\left[\begin{array}{cccc}
1 & 0 & 0 & 0 \\
-1 & 0 & 1 & 0 \\
0 & -1 & 0 & 0 \\
0 & 0 & 0 & 1
\end{array}\right] \text { and } \Re=\left[\begin{array}{cccc}
1 & 0 & 0 & 0 \\
0 & 1 & -1 & 0 \\
0 & 0 & 1 & 0 \\
0 & 0 & 0 & 1
\end{array}\right]
$$

then

$$
\mathbb{Q}_{0}^{*}=\pi \mathbb{Q}_{0}^{(2)} \Re=\left[\begin{array}{c:c}
I_{2} & \theta \\
\hdashline \theta & \theta
\end{array}\right]
$$

and

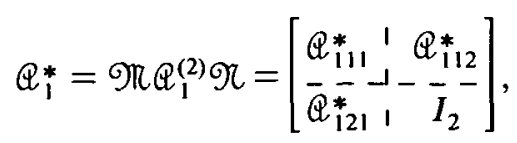


and hence the conditions of Theorem 3 apply to the finite sequence $\left\{\mathbb{Q}_{j}^{(2)}\right\}$. Thus we define

$$
\begin{aligned}
& \mathcal{L}=\left[\begin{array}{ccc}
I_{2} & 1 & \theta \\
\hdashline \mathbb{Q}_{112}^{*} & -1 & -I_{2}
\end{array}\right], \quad \mathscr{Q}=\left[\begin{array}{cc}
\theta^{1} & \mathbb{Q}_{121}^{*} \\
\hdashline & \perp \\
\hdashline \mathbb{Q}_{111}^{*}
\end{array}\right], \\
& W_{j}^{*(2)}=\left[\begin{array}{c:c}
\mathcal{X}_{j 21}^{*} & \mathcal{X}_{j 22}^{*} \\
\hdashline \mathcal{X}_{j+1,11}^{*} & \mathcal{X}_{j+1,12}^{*}
\end{array}\right] \text { and } \mathscr{K}=\left[\begin{array}{ccc}
\theta & I_{2} \\
\hdashline I_{2} & -\frac{\theta}{\theta}
\end{array}\right] \text {, }
\end{aligned}
$$

and obtain the "solution"

$$
\mathscr{W}_{j}^{*(2)}=(-1)^{j}\left(\mathcal{L}^{-1} \mathcal{Q}\right)^{j} \mathcal{E}^{-1} \mathscr{K} \quad \text { for all } j=0,1,2, \ldots
$$

Now we also know that the matrix $\mathscr{P}=(-1) \mathcal{L}^{-1} \mathscr{Q}$ satisfies the polynomial equation $\mathscr{P}^{3}=-\mathscr{P}^{2}-\mathscr{P}$ and hence it follows that

$$
\mathscr{w}_{j+3}^{*(2)}=-\mathscr{w}_{j}^{*(2)}-\mathscr{w}_{j+1}^{*(2)} \text { for all } j=0,1,2, \ldots
$$

It can now be seen that

$$
X_{j+8}=-X_{j+6}-X_{j+4} \text { for all } j=0,1,2, \ldots
$$

Since we can determine $X_{0}, X_{1}, \ldots, X_{7}$ directly by solving the equations

$$
\left[\begin{array}{cccccc}
A_{0} & \theta & \theta & \theta & \cdots & \theta \\
A_{1} & A_{0} & \theta & \theta & \cdots & \theta \\
A_{2} & A_{1} & A_{0} & \theta & \cdots & \theta \\
\theta & A_{2} & A_{1} & A_{0} & \cdots & \theta \\
\vdots & \vdots & \vdots & \vdots & & \vdots \\
\theta & \theta & \theta & \theta & \cdots & A_{0}
\end{array}\right]\left[\begin{array}{c}
X_{0} \\
X_{1} \\
X_{2} \\
X_{3} \\
\vdots \\
X_{9}
\end{array}\right]=\left[\begin{array}{c}
\theta \\
\theta \\
I_{2} \\
\theta \\
\vdots \\
\theta
\end{array}\right]
$$

we can now write down the complete solution.

\section{Input retrieval using power series inversion}

In this section we give a brief outline of how the power series inversion could be applied to input retrieval. We consider the system described by equations (1)-(6). The coefficients $J_{j}$ satisfy a recurrence relation of the form

$$
J_{j+r+1}=\sum_{k=1}^{r+1} \alpha_{k} J_{j+r+1-k} \text { for } j=0,1,2, \ldots,
$$


where $p(s)=s^{r+1}-\sum_{k=1}^{r+1} \alpha_{k} s^{r+1-k}$ is the minimum polynomial of the matrix $A$. Thus if we define

$$
J_{0}^{*}=J_{0} \quad \text { and } \quad J_{j}^{*}=J_{j}-\sum_{k=1}^{j} \alpha_{k} J_{j-k} \quad \text { for } j=0,1,2, \ldots, r,
$$

then we have

$$
\begin{aligned}
\left(p(s) / s^{r+1}\right) \cdot J(1 / s) & =\left(p(s) / s^{r+1}\right) \cdot \sum_{j=0}^{\infty} J_{j} / s^{j} \\
& =\sum_{j=0}^{r} J_{j}^{*} / s^{j}=J^{*}(1 / s) .
\end{aligned}
$$

If we assume now that $J_{0}^{*}\left(=J_{0}\right)$ is non singular then $J^{*}(1 / s)$ can be inverted by the method used in Theorem 2 . Therefore we define $\mathcal{L}=\mathscr{g}_{0}^{*(r)}$ and $\mathcal{U}=g_{1}^{*(r)}$. If we use the notation $K^{*}(1 / s)=\left[J^{*}(1 / s)\right]^{-1}$ and write

$$
K^{*}(1 / s)=\sum_{j=0}^{\infty} K_{j}^{*} / s^{j}
$$

then the coefficients $K_{j}^{*}$ satisfy a finite recurrence of the form

$$
K_{j+h r}^{*}=\sum_{k=1}^{h} \beta_{k} K_{j+h r-k r}^{*} \text { for } j=0,1,2, \ldots
$$

where $q(s)=s^{h}-\sum_{k=1}^{h} \beta_{k} s^{h-k}$ is the minimum polynomial of the matrix $(-1) \mathfrak{L}^{-1} \mathscr{Q}$. Thus we have

$$
\left(q\left(s^{r}\right) / s^{h r}\right) K^{*}(1 / s)=Q(1 / s)
$$

where $Q(1 / s)$ is a matrix polynomial of degree at most $(h r-1)$ in $1 / s$. It follows that $K(1 / s)=[J(1 / s)]^{-1}$ can be written as

$$
K(1 / s)=\left(p(s) / s^{r+1}\right) K^{*}(1 / s)=\frac{\left(p(s) / s^{r+1}\right) Q(1 / s)}{q\left(s^{r}\right) / s^{h r}}=\frac{P(1 / s)}{q\left(s^{r}\right) / s^{h r}}
$$

where $P(1 / s)$ is a polynomial of degree at most $(h+1) r$ in $1 / s$. Thus if we write

$$
K(1 / s)=\sum_{j=0}^{\infty} K_{j} / s^{j}
$$

it can be seen that the coefficients $K_{j}$ also satisfy a recurrence defined by the polynomial $q(s)$. In fact we have

$$
K_{j+r h}=\sum_{k=1}^{h} \beta_{k} K_{j+r h-r k} \quad \text { for } j=r+1, r+2, \ldots
$$

Because of this recurrence we can solve directly for the coefficients $K_{0}$, $K_{1}, \ldots, K_{r(h+1)}$ and hence determine the complete sequence $\left\{K_{j}\right\}$. 
Unfortunately the method as such is not very practical because the recurrence for the coefficients $K_{j}$ is not the simplest such recurrence. Before applying the suggested procedure we look therefore at an alternative method of inversion that does produce the simplest recurrence. This means that $\left\{K_{j}\right\}$ can be determined as above but with a much reduced finite system to solve for initial coefficients.

\section{A closed formula for inversion of the transfer function}

In this section we derive a closed formula for $[T(s)]^{-1}$. Following Rosenbrock [2] the system is represented by a single matrix. By performing a sequence of elementary row and column operations we obtain an alternative system matrix and relationship of the two matrices yields the key formula.

Before implementing the full inversion it is convenient to consider an important preliminary application. We define non singular square matrices $B^{\dagger}$ and $C^{\dagger}$ written in partitioned form as

$$
B^{\dagger}=\left[\begin{array}{l}
B_{1}^{\dagger} \\
B_{2}^{\dagger}
\end{array}\right] \text { and } C^{\dagger}=\left[\begin{array}{ll}
C_{1}^{\dagger} & C_{2}^{\dagger}
\end{array}\right]
$$

such that

$$
B^{\dagger} B=\left[\begin{array}{c}
I_{m} \\
\theta
\end{array}\right] \text { and } C C^{\dagger}=\left[\begin{array}{ll}
I_{m} & \theta
\end{array}\right] .
$$

These matrices can easily be constructed using elementary row operations but we will show later a specific method of construction. We now claim that the matrix

$$
N=\left[\begin{array}{c:c}
\theta & -C \\
\hdashline-B & I_{n}
\end{array}\right]
$$

can be converted using elementary row and column operations into each of the equivalent matrices $N_{1}$ and $N_{2}$ defined by

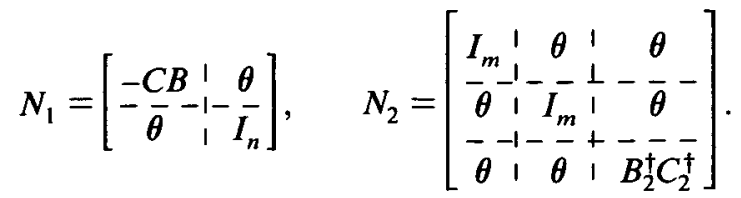

In fact we can convert $N_{1}$ into $N_{2}$ using left multiplication by

$$
L=\left[\begin{array}{c:cc}
\theta & B_{1}^{\dagger} \\
\hdashline I_{m} & -\bar{C} \\
\hdashline-B_{2}^{\dagger} C_{1}^{\dagger} & B_{2}^{\dagger}\left(I_{n}-C_{1}^{\dagger} C\right)
\end{array}\right]
$$


and right multiplication by

$$
R=\left[\begin{array}{c:c:c}
I_{m} & -B_{1}^{\dagger} C_{1}^{\dagger} & -B \dagger C_{2}^{\dagger} \\
\hdashline B & \left(I_{n}-B B_{1}^{\dagger}\right) C_{1}^{\dagger} & \left(I_{n}-B B_{1}^{\dagger}\right) C_{2}^{\dagger}
\end{array}\right] .
$$

Now since $N_{2}=L N_{1} R$ where $L$ and $R$ are non singular it follows that $N_{1}$ is invertible if and only if $N_{2}$ is invertible. Equivalently $(C B)^{-1}$ exists if and only if $\left(B_{2}^{\dagger} C_{2}^{\dagger}\right)^{-1}$ exists and when these inverses do exist

$$
N_{1}^{-1}=\left[\begin{array}{c:cc}
-(C B)^{-1} & & \theta \\
\hdashline \theta & - & I_{n}
\end{array}\right] \quad \text { and } \quad N_{2}^{-1}=\left[\begin{array}{c:c:cc}
I_{m} & \theta & \theta \\
\hdashline \theta & I_{m} & -\theta \\
\hdashline \theta & -\theta & \left(B_{2}^{\dagger} C_{2}^{\dagger}\right)^{-1}
\end{array}\right] .
$$

However we also know that $N_{1}^{-1}=R N_{2}^{-1} L$ and this gives

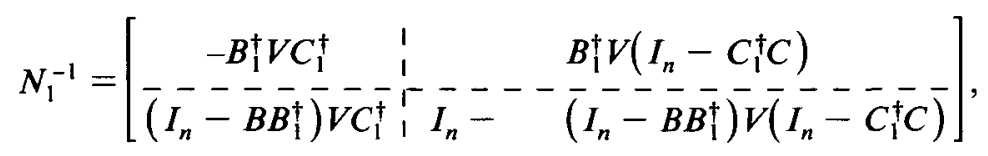

where $V=I_{n}-C_{2}^{\dagger}\left(B_{2}^{\dagger} C_{2}^{\dagger}\right)^{-1} B_{2}^{\dagger}$. From the original expression for $N_{1}^{-1}$ we therefore obtain

$$
(C B)^{-1}=B_{1}^{\dagger} V C_{1}^{\dagger}, \quad\left(I_{n}-B B_{1}^{\dagger}\right) V=\theta \quad \text { and } \quad V\left(I_{n}-C_{1}^{\dagger} C\right)=\theta .
$$

These formulae relate to subsequent inversion theorems.

A slightly modified version of the Rosenbrock system matrix can be written as

$$
N(s)=\left[\begin{array}{c:cc}
\theta & -C \\
\hdashline-B & s I_{n}^{-}-\bar{A}
\end{array}\right]
$$

and we can show that this matrix can be converted using elementary row and column operations into each of the equivalent matrices

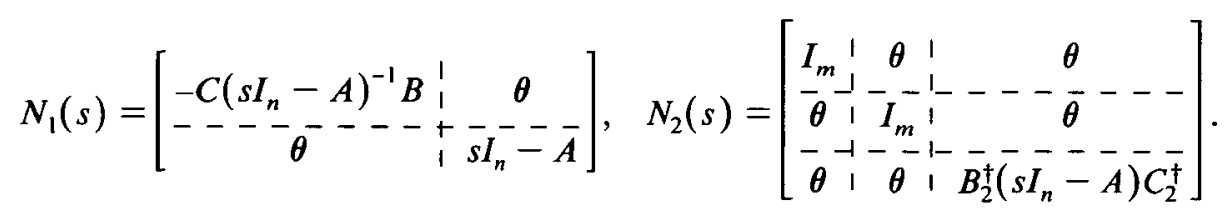

Conversion from $N_{1}(s)$ to $N_{2}(s)$ can be achieved using multiplication on the left by

$$
L(s)=\left[\begin{array}{c:c}
\theta & B_{1}^{\dagger} \\
\hdashline I_{m} & C\left(s I_{n}-A\right)^{-1} \\
\hdashline-B_{2}^{\dagger}\left(s I_{n}-A\right) C_{1}^{\dagger} & B_{2}^{\dagger}\left(s I_{n}-A\right)\left(I_{n}-C_{1}^{\dagger} C\right)\left(s I_{n}-A\right)^{-1}
\end{array}\right]
$$


and multiplication on the right by

$$
\begin{aligned}
& R(s)=\left[\begin{array}{c:c}
I_{m} & B_{1}^{\dagger}\left(s I_{n}-A\right) C_{1}^{\dagger} \\
\hdashline\left(s I_{n}-A\right)^{-1} B & \left(s I_{n}-A\right)^{-1}\left(I_{n}-B B_{1}^{\dagger}\right)\left(s I_{n}-A\right) C_{1}^{\dagger}
\end{array}\right. \\
& \left.\begin{array}{c}
B_{1}^{\dagger}\left(s I_{n}-A\right) C_{2}^{\dagger} \\
\left(s I_{n}-A\right)^{-1}\left(I_{n}-B B_{1}^{\dagger}\right)\left(s I_{n}-A\right) C_{2}^{\dagger}
\end{array}\right]
\end{aligned}
$$

We note that $L(s)$ and $R(s)$ are both non singular with determinants that do not depend on $s$. Since $N_{2}(s)=L(s) N_{1}(s) R(s)$ we can now see that $N_{1}(s)$ is invertible if and only if $N_{2}(s)$ is invertible. Equivalently, $\left[C\left(s I_{n}-A\right)^{-1} B\right]^{-1}$ exists if and only if $\left[B_{2}^{\dagger}\left(s I_{n}-A\right) C_{2}^{\dagger}\right]^{-1}$ exists in which case

$$
\left[N_{1}(s)\right]^{-1}=\left[\begin{array}{c:c}
-\left[C\left(s I_{n}-A\right)^{-1} B\right]^{-1} & \theta \\
\hdashline \theta & \left(s I_{n}-A\right)^{-1}
\end{array}\right]
$$

and

$$
\left[N_{2}(s)\right]^{-1}=\left[\begin{array}{c:c:c}
I_{m} & \theta & \theta \\
\hdashline \theta & I_{m} & \theta \\
\hdashline \theta & \theta & {\left[B_{2}^{\dagger}\left(s I_{n}-A\right) C_{2}^{\dagger}\right]^{-1}}
\end{array}\right]
$$

Since $\left[N_{1}(s)\right]^{-1}=R(s)\left[N_{2}(s)\right]^{-1} L(s)$ we also have

$$
\begin{aligned}
& {\left[N_{1}(s)\right]^{-1}=\left[\begin{array}{l}
-B_{1}^{\dagger} V(s) C_{-}^{\dagger} \\
\hdashline\left(s I_{n}-A\right)^{-1}\left(I_{n}-B B_{1}^{\dagger}\right) \bar{V}(s) C_{1}^{\dagger}
\end{array}\right.} \\
& \left.\begin{array}{c}
B_{1}^{\dagger} V(s)\left(I_{n}-C_{1}^{\dagger} C\right)\left(s I_{n}-A\right)^{-1} \\
\left(s I_{n}-A\right)^{-1}-\left(s I_{n}-A\right)^{-1}\left(I_{n}-B B_{1}^{\dagger}\right) V(s)\left(I_{n}-C_{1}^{\dagger} C\right)\left(s I_{n}-A\right)^{-1}
\end{array}\right]
\end{aligned}
$$

where $V(s)=\left(s I_{n}-A\right)\left\{\left(s I_{n}-A\right)^{-1}-C_{2}^{\dagger}\left[B B_{2}^{\dagger}\left(s I_{n}-A\right) C_{2}^{\dagger}\right]^{-1} B_{2}^{\dagger}\right\}\left(s I_{n}-A\right)$. From the original expression for $\left[N_{1}(s)\right]^{-1}$ we can now deduce that

$$
\left[C\left(s I_{n}-A\right)^{-1} B\right]^{-1}=B_{1}^{\dagger} V(s) C_{1}^{\dagger}, \quad\left(I_{n}-B B_{1}^{\dagger}\right) V(s)=\theta,
$$

and

$$
V(s)\left(I_{n}-C_{1}^{\dagger} C\right)=\theta .
$$

Thus we can state the following theorem.

THEOREM 7. The inverse transfer function $[T(s)]^{-1}=\left[C\left(s I_{n}-A\right)^{-1} B\right]^{-1}$ is well defined if and only if the matrix $\left[B_{2}^{\dagger}\left(s I_{n}-A\right) C_{2}^{\dagger}\right]^{-1}$ is well defined and in this case $[T(s)]^{-1}=B_{1}^{\dagger} V(s) C_{1}^{\dagger}$. 


\section{A formula for input retrieval-non singular case}

In this section we consider special formulae for input retrieval when $J_{0}=C B$ is non singular.

TheOREM 8. If $J_{0}^{-1}=(C B)^{-1}$ is well defined then $\operatorname{det}\left[B_{2}^{\dagger}\left(s I_{n}-A\right) C_{2}^{\dagger}\right]$ is a polynomial of degree $n-m$ with at most $n-m$ distinct zeros in the $s$ plane. At all other points $[T(s)]^{-1}=\left[C\left(s I_{n}-A\right)^{-1} B\right]^{-1}$ is well defined and

$$
[T(s)]^{-1}=\left[B_{1}^{\dagger} V C_{1}^{\dagger}\right] s-B_{1}^{\dagger} V A V C_{1}^{\dagger}-B_{1}^{\dagger} V A C_{2}^{\dagger}\left[B_{2}^{\dagger}\left(s I_{n}-A\right) C_{2}^{\dagger}\right]^{-1} B_{2}^{\dagger} A V C_{1}^{\dagger} \text {. }
$$

Proof. We have already noted that $\left(B_{2}^{\dagger} C_{2}^{\dagger}\right)^{-1}$ exists if and only if $(C B)^{-1}$ exists and hence $V=I_{n}-C_{2}^{\dagger}\left(B_{2}^{\dagger} C_{2}^{\dagger}\right)^{-1} B_{2}^{\dagger}$ is well defined. The result now follows by rearranging the expression for $V(s)$ in the equation $[T(s)]^{-1}=B_{\dagger}^{\dagger} V(s) C_{1}^{\dagger}$.

If we define $K_{0}=B_{1}^{\dagger} V C_{1}^{\dagger}, K_{1}=-B_{1}^{\dagger} V A V C_{1}^{\dagger}, \quad F=\left(B_{2}^{\dagger} C_{2}^{\dagger}\right)^{-1} B_{2}^{\dagger} A C_{2}^{\dagger}, \quad G=$ $\left(B_{2}^{\dagger} C_{2}^{\dagger}\right)^{-1} B_{2}^{\dagger} A V C_{1}^{\dagger}$ and $H=-B_{1}^{\dagger} V A C_{2}^{\dagger}$ then we can rewrite (21) as

$$
[T(s)]^{-1}=K_{0} s+K_{1}+H\left(s I_{n-m}-F\right)^{-1} G
$$

and hence equation (4) can be written as

$$
\bar{u}(s)=\left\{R(s)+H\left(s I_{n-m}-F\right)^{-1} G\right\} \bar{y}(s)
$$

where $R(s)=K_{0} s+K_{1}$. Therefore the input can be retrieved from a knowledge of the output only. By considering the "inverse" system

$$
\begin{aligned}
\dot{z}(t) & =F z(t)+G y(t) \\
v(t) & =H z(t)
\end{aligned}
$$

with $z(0)=\theta$ and where $y \in R^{m}$ is the input (the output of the original system (1)), $v \in R^{m}$ is the output and $z \in R^{n-m}$ is the state of the inverse system then we can retrieve the input to the original system (1) in the form

$$
u(t)=R(D) y(t)+v(t)
$$

where $R(D)=K_{0} D+K_{1}$ is a first order differential operator with constant coefficients.

Corollary 5. If $J_{0}^{-1}=(C B)^{-1}$ exists then we can write the inverse transfer function in the form

$$
[T(s)]^{-1}=s K(1 / s)=s \sum_{j=0}^{\infty} K_{j} / s^{j}
$$


for sufficiently large $s$, and where the coefficients $K_{j}$ satisfy a recurrence relation of the form

$$
K_{j+r}=\sum_{k=1}^{r} \beta_{k} K_{j+r-k}
$$

for all $j \geqslant 2$ where $p(s)=s^{r}-\sum_{k=1}^{r} \beta_{k} s^{r-k}$ is the minimum polynomial of the matrix $F=\left(B_{2}^{\dagger} C_{2}^{\dagger}\right)^{-1} B_{2}^{\dagger} A C_{2}^{\dagger}$.

Proof. Using the Neumann expansion in (22) for $\left(s I_{n-m}-F\right)^{-1}$ we obtain $K_{j}=H F^{j-2} G$ for all $j \geqslant 2$ from which the result follows.

When $J_{0}^{-1}=(C B)^{-1}$ is well defined there is a standard formula for constructing $B^{\dagger}$ and $C^{\dagger}$. Let $\mathscr{P}=\left\{p \mid p=\left(p_{1}, p_{2}, \ldots, p_{m}\right)\right\}$ where $p_{i}$ are integers such that $1 \leqslant p_{1}<p_{2}<\cdots p_{m} \leqslant n$ and for each $p \in \mathscr{P}$ define square matrices $B_{p}$ and $C_{p}$ by

$$
B_{p}=\left[\begin{array}{c}
b_{p_{1}}^{\prime} \\
b_{p_{2}}^{\prime} \\
\vdots \\
b_{p_{m}}^{\prime}
\end{array}\right] \text { and } C=\left[c_{p_{1}} c_{p_{2}} \cdots c_{p_{m}}\right]
$$

where $b_{i}^{\prime}$ is the $i$ th row of $B$ and $c_{j}$ is the $j$ th column of $C$. It was shown by Cauchy \& Binet in 1812 that

$$
\operatorname{det}(C B)=\sum_{p \in \mathcal{P}} \operatorname{det}\left(C_{p} B_{p}\right)
$$

Since $\operatorname{det}(C B) \neq 0$ there is at least one value of $p \in \mathscr{P}$ for which $\operatorname{det}\left(C_{p} B_{p}\right) \neq 0$ and for which therefore $B_{p}$ and $C_{p}$ are non singular. By reordering the components in the state vector of the system (1) we can assume that $p=(1,2, \ldots, m)$ in which case we can write

$$
B=\left[\begin{array}{c}
B_{1} \\
\hdashline B_{2}
\end{array}\right] \text { and } C=\left[\begin{array}{l:l}
C_{1} & C_{2}
\end{array}\right]
$$

where $B_{1}=B_{(1,2, \ldots, m)}$ and $C_{1}=C_{(1,2, \ldots, m)}$ are each non singular. Now we can define

$$
B^{\dagger}=\left[\begin{array}{c}
B_{1}^{\dagger} \\
\hdashline B_{2}^{\dagger}
\end{array}\right]=\left[\begin{array}{c:c}
B_{1}^{-1} & \theta \\
\hdashline-B_{2} B_{1}^{-1} & I_{n-m}^{-}
\end{array}\right] \text {and } C^{\dagger}=\left[C_{1}^{\dagger} C_{2}^{\dagger}\right]=\left[\begin{array}{ccc}
C^{-1} & -C_{1}^{-1} C_{2} \\
\hdashline \theta & I_{n-m}
\end{array}\right] \text {. }
$$

It is straightforward to show that

$$
B_{2}^{\dagger} C_{2}^{\dagger}=I_{n-m}+B_{2}\left(C_{1} B_{1}\right)^{-1} C_{2},
$$


and

$$
\left(B_{2}^{\dagger} C_{2}^{\dagger}\right)^{-1}=I_{n-m}-B_{2}(C B)^{-1} C_{2} \text {. }
$$

EXAMPLE. We now illustrate previous results with a numerical example. For the system described by equation (1) take

$$
A=\left[\begin{array}{ccccc}
1 & 0 & 0 & 0 & 0 \\
0 & -1 & 0 & 0 & 0 \\
0 & 0 & 2 & 0 & 0 \\
0 & 0 & 0 & 3 & 0 \\
0 & 0 & 0 & 0 & -2
\end{array}\right], \quad B=\left[\begin{array}{ccc}
1 & 0 & 0 \\
0 & 1 & 0 \\
1 & -1 & 0 \\
1 & 2 & 0 \\
0 & 0 & 1
\end{array}\right]
$$

and

$$
C=\left[\begin{array}{ccccc}
1 & 2 & 0 & -3 & 0 \\
0 & 0 & 1 & 1 & 0 \\
0 & 0 & 0 & 0 & 1
\end{array}\right] .
$$

A suitable choice of $p \in \mathscr{P}$ (as defined above) is $p=(1,3,5)$ and hence we reorder the components of the state which gives

$$
A=\left[\begin{array}{ccccc}
1 & 0 & 0 & 0 & 0 \\
0 & 2 & 0 & 0 & 0 \\
0 & 0 & -2 & 0 & 0 \\
0 & 0 & 0 & -1 & 0 \\
0 & 0 & 0 & 0 & 3
\end{array}\right], \quad B=\left[\begin{array}{ccc}
1 & 0 & 0 \\
1 & -1 & 0 \\
0 & 0 & 1 \\
\hdashline 0 & 1 & 0 \\
1 & 2 & 0
\end{array}\right]
$$

and

$$
C=\left[\begin{array}{ccc:cc}
1 & 0 & 0 & 2 & -3 \\
0 & 1 & 0 & 0 & 1 \\
0 & 0 & 1 & 0 & 0
\end{array}\right]
$$

Thus we have

$$
B^{\dagger}=\left[\begin{array}{ccc:cc}
1 & 0 & 0 & 0 & 0 \\
1 & -1 & 0 & 0 & 0 \\
0 & 0 & 1 & 0 & 0 \\
\hdashline-1 & 1 & 0 & 1 & 0 \\
-3 & 2 & 0 & 0 & 1
\end{array}\right] \quad \text { and } \quad C^{\dagger}=\left[\begin{array}{ccc:cc}
1 & 0 & 0 & -2 & 3 \\
0 & 1 & 0 & 0 & -1 \\
0 & 0 & 1 & 0 & 0 \\
\hdashline 0 & 0 & 0 & 1 & 0 \\
0 & 0 & 0 & 0 & 1
\end{array}\right]
$$

We can then use the formulae of Section 6 to calculate

$$
\begin{gathered}
K_{0}=\left[\begin{array}{ccc}
1 / 6 & 2 / 3 & 0 \\
-1 / 3 & -1 / 3 & 0 \\
0 & 0 & 1
\end{array}\right], \quad K_{1}=\left[\begin{array}{ccc}
-5 / 9 & -14 / 9 & 0 \\
29 / 18 & 10 / 9 & 0 \\
0 & 0 & 2
\end{array}\right], \\
F=\left[\begin{array}{cc}
-7 / 3 & -5 / 3 \\
-2 & 0
\end{array}\right], \quad G=\left[\begin{array}{ccc}
35 / 18 & 13 / 9 & 0 \\
7 / 6 & 2 / 3 & 0
\end{array}\right] \text { and } H=\left[\begin{array}{cc}
2 / 3 & 1 / 3 \\
0 & 0 \\
-4 / 3 & -5 / 3
\end{array}\right] .
\end{gathered}
$$


Since the characteristic polynomial of $F$ is $s^{2}+(7 / 3) s-10 / 3$ the coefficients $K_{j}$ are defined by $K_{0}, K_{1}$ and the recurrence relation

$$
K_{j+2}=-\frac{7}{3} K_{j+1}+\frac{10}{3} K_{j}
$$

for $j=0,1,2, \ldots$. If we wish only to determine the coefficients $K_{j}$ then an alternative procedure is to calculate $\operatorname{det}\left[B_{2}^{\dagger}\left(s I_{n}-A\right) C_{2}^{\dagger}\right]$ (which is essentially the characteristic polynomial of $F$ and hence determines the above recurrence) and then find $K_{0}$ and $K_{1}$ simply by solving the equation

$$
\left[\begin{array}{ll}
J_{0} & \theta \\
J_{1} & J_{0}
\end{array}\right]\left[\begin{array}{c}
K_{0} \\
K_{1}
\end{array}\right]=\left[\begin{array}{c}
I_{3} \\
\theta
\end{array}\right]
$$

\section{A formula for input retrieval-singular case}

In this section we consider special formulae for input retrieval when $J_{0}=C B$ is singular. We use the general matrix inversion formula to write

$$
\left[B_{2}^{\dagger}\left(s I_{n}-A\right) C_{2}^{\dagger}\right]^{-1}=\frac{\operatorname{adj}\left[B_{2}^{\dagger}\left(s I_{n}-A\right) C_{2}^{\dagger}\right]}{\operatorname{det}\left[B_{2}^{\dagger}\left(s I_{n}-A\right) C_{2}^{\dagger}\right]}
$$

Now because $B_{2}^{\dagger} C_{2}^{\dagger}$ is singular we can find an integer $h$ with $0 \leqslant h \leqslant n-m-1$ such that the previous expression can be rewritten in the form

$$
\left[B_{2}^{\dagger}\left(s I_{n}-A\right) C_{2}^{\dagger}\right]^{-1}=Q(s) / q(s),
$$

where $Q(s)$ is a matrix polynomial of degree at most $n-m-1$ and where $q(s)=s^{h}-\sum_{j=1}^{h} \beta_{j} s^{h-j}$ is a scalar polynomial of degree $h$. Now from Theorem 7 we obtain

$$
[T(s)]^{-1}=\left[q(s) B_{1}^{\dagger}\left(s I_{n}-A\right) C_{1}^{\dagger}-B_{1}^{\dagger}\left(s I_{n}-A\right) C_{2}^{\dagger} Q(s) B_{2}^{\dagger}\left(s I_{n}-A\right) C_{1}^{\dagger}\right] / q(s)
$$

and since the numerator is a matrix polynomial of degree at most $n-m+1$ it is easily seen that we can write

$$
[T(s)]^{-1}=s^{n-m+1-h} \sum_{j=0}^{\infty} K_{j} / s^{j} .
$$


By using the previous expression to equate coefficients it can be seen that the coefficients $K_{j}$ satisfy a recurrence relation of the form

$$
K_{n-m+1+k}=\sum_{j=1}^{h} \beta_{j} K_{n-m+1+k-j}
$$

for each $k=1,2,3, \ldots$. Thus if we can find $K_{0}, K_{1}, \ldots, K_{n-m+1}$ directly we can determine all subsequent $K_{j}$ from the recurrence relation. From the expression

$$
T(s)=(1 / s) \sum_{j=0}^{\infty} J_{j} / s^{j},
$$

we can equate coefficients using (23) and (24) to see that

$$
\sum_{j=0}^{k} J_{k-j} K_{j}=\sum_{j=0}^{k} K_{j} J_{k-j}=\theta \text { for } k \neq i
$$

and

$$
\sum_{j=0}^{i} J_{i-j} K_{j}=\sum_{j=0}^{i} K_{j} J_{i-j}=I_{m}
$$

where we are writing $i=n-m-h$. From these equations it can be seen that the vector space generated by the rows of the matrices $J_{0}, J_{1}, \ldots, J_{i}$ is precisely $R^{m}$. Therefore if we assume $K_{0}, K_{1}, \ldots, K_{j-1}$ are known then $K_{j}$ is uniquely determined by the equations

$$
\left[\begin{array}{llll}
J_{0} & \theta & \cdots & \theta \\
J_{1} & J_{0} & \cdots & \theta \\
\vdots & \vdots & & \vdots \\
J_{i} & J_{i-1} & \cdots & J_{0}
\end{array}\right]\left[\begin{array}{l}
K_{j} \\
K_{j+1} \\
\vdots \\
K_{j+1}
\end{array}\right]=-\left[\begin{array}{llll}
J_{j} & J_{j-1} & \cdots & J_{1} \\
J_{j+1} & J_{j} & \cdots & J_{2} \\
\vdots & \vdots & & \vdots \\
J_{j+i} & J_{j+i-1} & \cdots & J_{i+1}
\end{array}\right]\left[\begin{array}{l}
K_{0} \\
K_{1} \\
\vdots \\
K_{j-1}
\end{array}\right] .
$$

Thus $K_{0}, K_{1}, \ldots, K_{i+h+1}$ are uniquely determined by the equations

$$
\sum_{j=0}^{k} J_{k-j} K_{j}=\theta \text { for } k \neq i, 0 \leqslant k \leqslant 2 i+h+1
$$

and

$$
\sum_{j=0}^{i} J_{i-j} K_{j}=I_{m}
$$


It is now possible to apply a standard realization procedure by setting

$$
\begin{gathered}
F=\left[\begin{array}{ccccc}
\theta & I_{m} & \theta & \cdots & \theta \\
\theta & \theta & I_{m} & \cdots & \theta \\
\vdots & \vdots & \vdots & & \vdots \\
\theta & \theta & \theta & \cdots & I_{m} \\
\beta_{h} I_{m} & \beta_{h-1} I_{m} & \beta_{h-2} I_{m} & \cdots & \beta_{1} I_{m}
\end{array}\right], \\
G=\left[\begin{array}{c}
K_{i+2} \\
K_{i+3} \\
\vdots \\
K_{i+h+1} \\
K_{i+h+2}
\end{array}\right] \text { and } H=\left[I_{m} \theta \theta \cdots \theta\right],
\end{gathered}
$$

where $F: R^{h m} \rightarrow R^{h m}, G: R^{m} \rightarrow R^{h m}$ and $H: R^{h m} \rightarrow R^{m}$. We see from this construction that

$$
H\left(s I_{h m}-F\right)^{-1} G=\sum_{j=i+2}^{\infty} K_{j} / s^{j-(i+1)},
$$

and hence we can now retrieve the input in the form

$$
\bar{u}(s)=\left\{\left(\sum_{j=0}^{i+1} K_{j} s^{(i+1)-j}\right)+H\left(s I_{h m}-F\right)^{-1} G\right\} \bar{y}(s) .
$$

Thus we see again that the input is retrieved from a knowledge of the output only. By considering the "inverse" system

$$
\begin{aligned}
& \dot{z}(t)=F z(t)+G y(t), \\
& v(t)=H z(t),
\end{aligned}
$$

with $z(0)=\theta$ and where $y \in R^{m}, v \in R^{m}$ and $z \in R^{h m}$ are the input, output and state of the system we can retrieve the input to the original system (1) in the form

$$
u(t)=R(D) y(t)+v(t)
$$

where $R(D)=\sum_{j=0}^{i+1} K_{j} D^{(i+1)-j}$ is a differential operator of order $(i+1)$ with constant coefficients. Of course this procedure can also be applied when $(C B)^{-1}$ exists.

EXAMPLE. We illustrate these results with the following example. Define

$$
A=\left[\begin{array}{cccc}
1 & 0 & 0 & 0 \\
0 & -1 & 0 & 0 \\
0 & 0 & 2 & 0 \\
0 & 0 & 0 & 2
\end{array}\right], \quad B=\left[\begin{array}{cc}
1 & 0 \\
0 & 1 \\
-2 & -2 \\
0 & -1
\end{array}\right] \text { and } \quad C=\left[\begin{array}{cccc}
1 & 0 & -1 & 2 \\
0 & 1 & 2 & -3
\end{array}\right] \text {. }
$$


It is easy to see that suitable matrices $B^{\dagger}$ and $C^{\dagger}$ are given by

$$
B^{\dagger}=\left[\begin{array}{c}
B_{1}^{\dagger} \\
\hdashline B_{2}^{\dagger}
\end{array}\right]=\left[\begin{array}{cccc}
1 & 0 & 0 & 0 \\
0 & -\frac{1}{2} & -\frac{0}{1} & -\frac{0}{0} \\
0 & 1 & 0 & 1
\end{array}\right], \quad C^{\dagger}=\left[\begin{array}{lll}
C_{1}^{\dagger} & C_{2}^{\dagger}
\end{array}\right]=\left[\begin{array}{cc:cc}
1 & 0 & 1 & -2 \\
0 & 1 & -2 & 3 \\
0 & 0 & 1 & 0 \\
0 & 0 & 0 & 1
\end{array}\right],
$$

and hence we have

$$
B_{2}^{\dagger} C_{2}^{\dagger}=\left[\begin{array}{ll}
-1 & 2 \\
-2 & 4
\end{array}\right], \quad B_{2}^{\dagger} A C_{2}^{\dagger}=\left[\begin{array}{cc}
8 & -10 \\
2 & -1
\end{array}\right] .
$$

Thus $\operatorname{det}\left[B_{2}^{\dagger}\left(s I_{4}-A\right) C_{2}^{\dagger}\right]=-9 s+12$ and the coefficients $K_{j}$ therefore satisfy the recurrence relation

$$
K_{j+1}=\frac{4}{3} K_{j}
$$

for each $j=3,4,5, \ldots$ and we must solve the equations

$$
\left[\begin{array}{lllll}
J_{0} & \theta & \theta & \theta & \theta \\
J_{1} & J_{0} & \theta & \theta & \theta \\
J_{2} & J_{1} & J_{0} & \theta & \theta \\
J_{3} & J_{2} & J_{1} & J_{0} & \theta \\
J_{4} & J_{3} & J_{2} & J_{1} & J_{0}
\end{array}\right]\left[\begin{array}{c}
K_{0} \\
K_{1} \\
K_{2} \\
K_{3} \\
K_{4}
\end{array}\right]=\left[\begin{array}{c}
\theta \\
I_{2} \\
\theta \\
\theta \\
\theta
\end{array}\right]
$$

to find, initially, $K_{0}, K_{1}, K_{2}$ and $K_{3}$. We obtain the solution

$$
\begin{array}{cc}
K_{0}=\left[\begin{array}{cc}
0 & 0 \\
-4 / 9 & -1 / 3
\end{array}\right], \quad K_{1}=\left[\begin{array}{cc}
1 / 3 & 0 \\
8 / 27 & 1 / 3
\end{array}\right], \\
K_{2}=\left[\begin{array}{cc}
-5 / 9 & 0 \\
68 / 81 & 2 / 3
\end{array}\right] \text { and } K_{3}=\left[\begin{array}{cc}
-2 / 27 & 0 \\
56 / 243 & 0
\end{array}\right] .
\end{array}
$$

Hence the "inverse" system is defined by the matrices

$$
F=\left[\begin{array}{cc}
4 / 3 & 0 \\
0 & 4 / 3
\end{array}\right], \quad G=\left[\begin{array}{cc}
-2 / 27 & 0 \\
56 / 243 & 0
\end{array}\right], \quad H=\left[\begin{array}{cc}
1 & 0 \\
0 & 1
\end{array}\right],
$$

and the full input retrieval in the form (26) as

$$
\begin{array}{r}
\bar{u}(s)=\left\{\left[\begin{array}{cc}
0 & 0 \\
-4 / 9 & -1 / 3
\end{array}\right] s^{2}+\left[\begin{array}{cc}
1 / 3 & 0 \\
8 / 27 & 1 / 3
\end{array}\right] s+\left[\begin{array}{cc}
-5 / 9 & 0 \\
68 / 81 & 2 / 3
\end{array}\right]\right. \\
\left.+\left[\begin{array}{cc}
s-4 / 3 & 0 \\
0 & s-4 / 3
\end{array}\right]^{-1}\left[\begin{array}{cc}
-2 / 27 & 0 \\
56 / 243 & 0
\end{array}\right]\right\} \bar{y}(s) .
\end{array}
$$




\section{Concluding remarks}

One of the principal reasons for the method of investigation used in this paper was a desire to extend input retrieval to infinite dimensional systems and one of the main problems in this regard was a lack of directly applicable inversion formulae for systems having the form of (1). It is known that some of the formulae in this paper can be applied to infinite dimensional systems, at least in certain circumstances, and further research will proceed in that direction.

\section{Appendix: a test to determine the inversion procedure}

In this section we will indicate how a rank test due to Sain and Massey [3] can be applied to determine the precise inversion procedure.

LEMMA 2. Let $\left\{A_{j}\right\}$ be a sequence of square matrices. If we define $\Delta(i)$ by

$$
\Delta(i)= \begin{cases}\operatorname{rank} \mathbb{Q}_{0}^{(i+1)}, & \text { if } i=0, \\ \operatorname{rank} \mathbb{Q}_{0}^{(i+1)}-\operatorname{rank} \mathbb{Q}_{0}^{(i)}, & \text { if } i=1,2,3, \ldots,\end{cases}
$$

we have $\Delta(i+1) \geqslant \Delta(i)$ for all $i=0,1,2, \ldots$.

This result is due to Sain and Massey and the proof is omitted.

THEOREM 9. Let $\left\{A_{j}\right\}$ be a sequence of square $(m \times m)$ matrices with $\Delta(i)$ as defined in Lemma 2. We can see that $A_{0}$ is non singular if and only if $\Delta(0)=m$. On the other hand when $\Delta(0)<m$ we can find non singular matrices $\mathscr{R}$ and $\Re$ such that

$$
\Re \mathbb{Q}_{0}^{(i)} \mathscr{K}=\left[\begin{array}{c:c}
I_{m_{1}} & \theta \\
\hdashline \theta & \theta
\end{array}\right] \text { and } \mathfrak{K} \mathbb{Q}_{1}^{(i)} \Re=\left[\begin{array}{c:c}
\mathcal{Q}_{111}^{*} & \mathcal{Q}_{112}^{*} \\
\hdashline \mathcal{Q}_{121}^{*} & I_{m_{2}}
\end{array}\right]
$$

where $m_{1}, m_{2}>0$ and $m_{1}+m_{2}=i m$, if and only if $\Delta(i)=m$.

Proof. The case where $A_{0}$ is non singular is obvious. Otherwise the conditions stated above are equivalent to

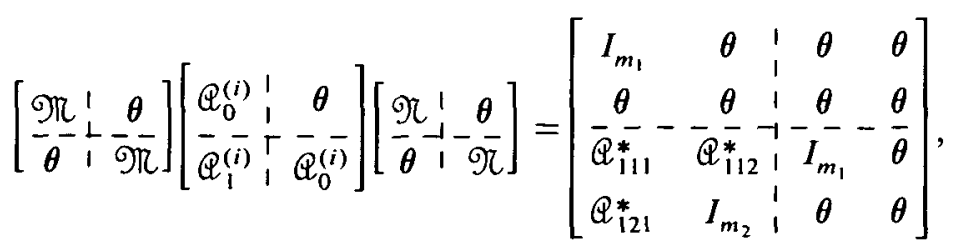


which is equivalent, in turn, to

$$
\operatorname{rank}\left[\begin{array}{c:c}
\mathbb{Q}_{0}^{(i)} & \theta \\
\hdashline \mathbb{Q}_{1}^{(i)} & \mathcal{Q}_{0}^{(i)}
\end{array}\right]=2 m_{1}+m_{2}=i m+m_{1} \quad \text { and } \operatorname{rank}\left(\mathbb{Q}_{0}^{(i)}\right)=m_{1},
$$

that is, $\operatorname{rank}\left(\mathbb{Q}_{0}^{(2 i)}\right)-\operatorname{rank}\left(\mathbb{Q}_{0}^{(i)}\right)=i m$. By Lemma 2 this condition is equivalent to $\Delta(i)=m$.

In order to determine which inversion procedure to use we simply calculate the rank of $Q_{0}^{(i)}$ for each value $i=1,2,3, \ldots$ until we find the first value of $i$ for which $\Delta(i)=m$. By Theorem 9 we can now determine which of the Theorems 1,3 or 5 to use in the inversion procedure.

\section{Acknowledgement}

I would like to thank Dr. Tony Pritchard, Director of the Control Theory Centre at the University of Warwick, U.K., who suggested the problem and gave valuable assistance to me during my visit to the Control Theory Centre in 1979, when much of this research was done.

\section{References}

[1] R. W. Brockett and M. D. Mesarovic, "The reproducibility of multivariable systems", J. Math. A nal. Appl. 11 (1965), 548-563.

[2] H. H. Rosenbrock, State space and multivariable theory (Nelson, London, 1970).

[3] M. K. Sain and J. L. Massey, "Invertibility of linear time invariant dynamical systems", IEEE Trans. Auto. Control AC-14 (1969), 141-149.

[4] L. M. Silverman, "Inversion of multivariable linear systems", IEEE Trans. Auto. Control AC-14 (1969), 270-276.

[5] S. H. Wang and E. J. Davison, "A new invertibility criterion for linear multivariable systems", IEEE Trans. Auto. Control (Tech. Notes and Corresp.) AC-18 (1973), 538-539.

[6] A. S. Willsky, "On the invertibility of linear systems", IEEE Trans. Auto. Control AC-19 (1974), 272-274.

School of Mathematics and Computer Studies

South Australian Institute of Technology

Ingle Farm

South Australia 5098 